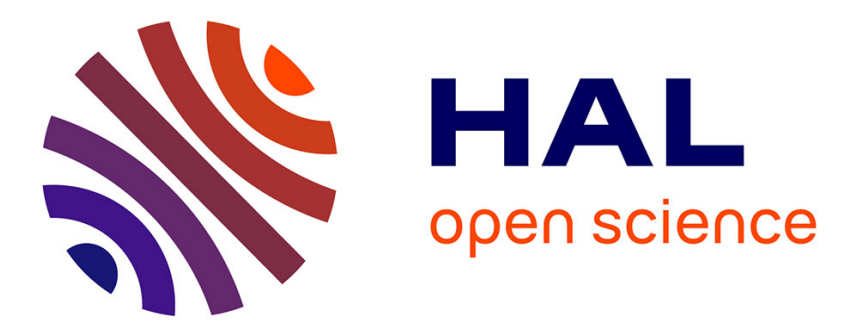

\title{
Fault Tolerant Control for Takagi-Sugeno systems with unmeasurable premise variables by trajectory tracking
}

Dalil Ichalal, Benoît Marx, José Ragot, Didier Maquin

\section{To cite this version:}

Dalil Ichalal, Benoît Marx, José Ragot, Didier Maquin. Fault Tolerant Control for Takagi-Sugeno systems with unmeasurable premise variables by trajectory tracking. IEEE International Symposium on Industrial Electronics, ISIE 2010, Jul 2010, Bari, Italy. pp.CDROM, 10.1109/ISIE.2010.5637766 . hal-00465573

\section{HAL Id: hal-00465573 \\ https://hal.science/hal-00465573}

Submitted on 9 Apr 2014

HAL is a multi-disciplinary open access archive for the deposit and dissemination of scientific research documents, whether they are published or not. The documents may come from teaching and research institutions in France or abroad, or from public or private research centers.
L'archive ouverte pluridisciplinaire HAL, est destinée au dépôt et à la diffusion de documents scientifiques de niveau recherche, publiés ou non, émanant des établissements d'enseignement et de recherche français ou étrangers, des laboratoires publics ou privés. 


\title{
Fault Tolerant Control for Takagi-Sugeno systems with unmeasurable premise variables by trajectory tracking
}

\author{
Dalil Ichalal, Benoit Marx, José Ragot, Didier Maquin
}

\begin{abstract}
This paper presents a new method for fault tolerant control of nonlinear systems described by TakagiSugeno fuzzy systems with unmeasurable premise variables. The idea is to use a reference model and design a new control law to minimize the state deviation between a healthy reference model and the eventually faulty actual model. This scheme requires the knowledge of the system states and of the occurring faults. These signals are estimated from a Proportional-Integral Observer (PIO) or Proportional-MultiIntegral Observer (PMIO). The fault tolerant control law is designed by using the Lyapunov method to obtain conditions which are given in Linear Matrix Inequality formulation (LMI). Finally, an example is included.
\end{abstract}

Index Terms- Takagi-Sugeno fuzzy systems, state and fault estimation, PI and PMI observers, Lyapunov stability analysis, linear matrix inequality.

\section{INTRODUCTION}

Fault tolerant control (FTC) has been recently introduced in the fault diagnosis framework. It consists to compute a new control law by taking into account the faults affecting the system in order to maintain acceptable performances and preserve stability of the system in the faulty situations.

The existing strategies are classified into two classes. The first class is called passive fault tolerant control or robust control. In this approach, the faults are treated as uncertainties. Therefore, the control is designed to be robust only to the specified faults. Contrarily to the passive FTC, active FTC requires a FDI block to detect, isolate and estimate the faults. The informations issued from the FDI block are used by the FTC module to reconfigure the control law in order to compensate the fault and ensure an acceptable system performances.

The active fault tolerant control has been developed essentially for linear systems [4], [15], [13], [11] and descriptor linear systems [9]. Clearly, linear models do not often represent accurately physical systems due to nonlinear behaviors. It is then interesting to work directly with nonlinear models. Nevertheless, from the mathematical point of view, working with nonlinear models is much harder than with linear ones. A new representation that combines simplicity of linear models and accuracy of nonlinear behaviors is introduced, initially, in [16] and known as Takagi-Sugeno (T-S) models. The idea is to consider a set of system operating points. At each operating point, the system is represented by a simple

All the authors are with the Centre de Recherche en Automatique de Nancy (CRAN), Nancy-Université, 2, avenue de la forêt de Haye 54516 Vandoeuvre-les-Nancy \{dalil.ichalal, benoit.marx, jose.ragot, didier.maquin\}eensem. inpl-nancy. fr linear sub-model. An interpolation of all these sub-models with nonlinear functions satisfying the sum convex property allows to obtain the global behavior of the system. One can cite some works in FTC field for nonlinear systems, for example, in [5], the authors give a method for actuator faults by using their estimations, for nonlinear descriptor systems with Lipschitz nonlinearities. In [14], a method requiring only the fault isolation is proposed for T-S systems. It is based on controller based observers bank. A switching system is then designed, to switch from one controller to an other, from the residual decision logic.

This paper is dedicated to the design of a fault tolerant control strategy for nonlinear systems described by TakagiSugeno models with unmeasurable premise variables. The main idea is to re-use the nominal control input developed in fault-free case to which two terms, related to the occurred fault and the tracking error trajectory between the system and a reference model, are added to be able to compensate the fault. The reference trajectory is provided by a reference model representing the system without faults. In addition, the control law requires the knowledge of the state of the system and faults affecting it. For that purpose, a PI (or PMI) observer is used to estimate simultaneously these signals.

The second section is dedicated to a brief presentation of Takagi-Sugeno models. The third section deals with the problem of fault tolerant control design with PI and PMI observers. Finally, an academic example is proposed in order to illustrate the FTC strategy.

\section{TAKAGI-SUGENO STRUCTURE FOR MODELING}

Consider a nonlinear system described by

$$
\left\{\begin{array}{l}
\dot{x}(t)=f(x(t), u(t)) \\
y(t)=h(x(t), u(t))
\end{array}\right.
$$

The T-S fuzzy modeling allows to represent the behavior of a nonlinear system (1) by the interpolation of a set of linear sub-models. Each sub-model contributes to the global behavior of the nonlinear system through a weighting function $\mu_{i}(\xi(t))$. The T-S structure is given by

$$
\left\{\begin{array}{l}
\dot{x}(t)=\sum_{i=1}^{r} \mu_{i}(\xi(t))\left(A_{i} x(t)+B_{i} u(t)\right) \\
y(t)=\sum_{i=1}^{r} \mu_{i}(\xi(t))\left(C_{i} x(t)+D_{i} u(t)\right)
\end{array}\right.
$$

where $x(t) \in \mathbb{R}^{n}$ is the state vector, $u(t) \in \mathbb{R}^{m}$ is the input vector, $y(t) \in \mathbb{R}^{p}$ represents the output vector. $A_{i} \in \mathbb{R}^{n \times n}$, $B_{i} \in \mathbb{R}^{n \times m}, C_{i} \in \mathbb{R}^{p \times n}$ and $D_{i} \in \mathbb{R}^{p \times m}$ are known matrices. The functions $\mu_{i}(\xi(t))$ are the weighting functions 
depending on the variables $\xi(t)$ which can be measurable (as the input or the output of the system) or non measurable variables (as the state of the system). These functions verify the following properties

$$
\left\{\begin{array}{l}
\sum_{i=1}^{r} \mu_{i}(\xi(t))=1 \\
0 \leq \mu_{i}(\xi(t)) \leq 1 \quad \forall i \in\{1,2, \ldots, r\}
\end{array}\right.
$$

Obtaining a T-S model (2) from (1) can be performed from different methods such us linearizing the system (1) around some operating points and using adequate weighting functions. It can also be obtained by black-box approaches which allow to identify the parameters of the model from inputoutput data. Finally, the most interesting and important way to obtain a model in the form (2) is the well-known nonlinear sector transformations [17], [12]. Indeed, this transformation allows to obtain an exact T-S representation of (1) with no information loss on a compact of state space.

Thanks to the convex sum property of the weighing functions (3), it is possible to generalize some tools developed in the linear domain to the nonlinear systems. This representation (2) is very interesting in the sense that it simplifies the stability studies of nonlinear systems and the design of control laws and observers. In [2], [6], [7], the stability and stabilization tools are inspired from the study of linear systems. In [1], [10], the authors worked on the problem of state estimation and diagnosis of T-S fuzzy systems. The proposed approaches in these last papers rely on the generalization of the classical observers (Luenberger Observer [8] and Unknown Input Observer (UIO) [3]) to the nonlinear domain. In the remaining of the paper, we use the following lemmas.

Lemma 1: Consider two matrices $X$ and $Y$ with appropriate dimensions and $\Omega$ a positive definite matrix. the following property is verified

$$
X^{T} Y+Y^{T} X \leq X^{T} \Omega X+Y^{T} \Omega^{-1} Y \quad \Omega>0
$$

Lemma 2: (Congruence) Let two matrices $P$ and $Q$, if $P$ is positive definite and if $Q$ is a full column rank matrix, than the matrix $Q P Q^{T}$ is positive definite.

\section{FAULT TOLERANT CONTROL OF T-S FUZZY SYSTEMS}

Let us consider the T-S reference model without faults described by (2). The system with faults $f$ is described by the following T-S model with unmeasurable premise variable

$$
\left\{\begin{array}{l}
\dot{x}_{f}(t)=\sum_{i=1}^{r} \mu_{i}\left(x_{f}(t)\right)\left(A_{i} x_{f}(t)+B_{i}\left(u_{f}(t)+f(t)\right)\right) \\
y_{f}(t)=C x_{f}(t)+R f(t)
\end{array}\right.
$$

For sake of simplicity, the time variable is omitted.

The goal is to design the control law $u_{f}(t)$ such that the system state $x_{f}(t)$ converges toward the reference state $x(t)$ given by the reference model (2). The control strategy is illustrated in the figure 1 . The following structure is proposed for the control law

$$
u_{f}=\sum_{i=1}^{r} \mu_{i}\left(\hat{x}_{f}\right)\left(-\hat{f}+K_{1 i}\left(x-\hat{x}_{f}\right)+u\right)
$$

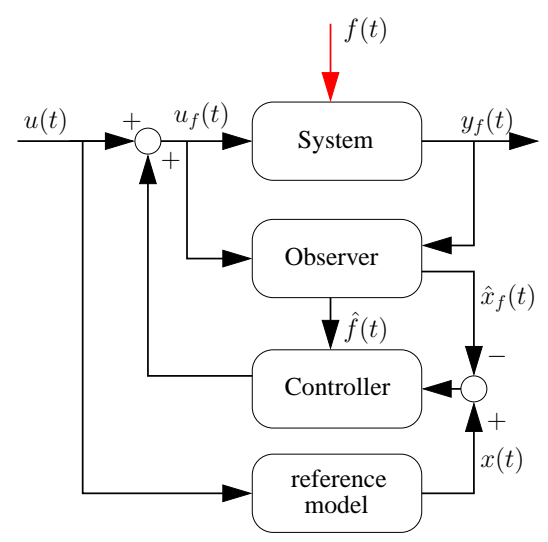

Fig. 1. Fault tolerant control scheme

The matrices $K_{1 i}$ are determined in order to ensure the stability of the system even if faults occur and to minimize the difference between $x_{f}(t)$ and $x(t)$. By analyzing the structure of $u_{f}(t)$ given in equation (6), the estimation of the state $x_{f}(t)$ and the $f(t)$ faults is required. This task is performed via a Proportional-Integral observer which estimates simultaneously the state and the faults of the system.

Let us consider the PI observer

$$
\begin{aligned}
\dot{\hat{x}}_{f} & =\sum_{i=1}^{r} \mu_{i}\left(\hat{x}_{f}\right)\left(A_{i} \hat{x}_{f}+B_{i}\left(u_{f}+\hat{f}\right)\right. \\
& \left.+H_{1 i}\left(y_{f}-\hat{y}_{f}\right)\right) \\
\dot{\hat{f}} & =\sum_{i=1}^{r} \mu_{i}\left(\hat{x}_{f}\right)\left(H_{2 i}\left(y_{f}-\hat{y}_{f}\right)\right) \\
\hat{y}_{f} & =C \hat{x}_{f}+R \hat{f}
\end{aligned}
$$

which depends on the gains $H_{1 i}$ and $H_{2 i}$.

The output error between the system (5) and the observer (7)-(8) is written by

$$
y_{f}-\hat{y}_{f}=\tilde{C} e_{a}
$$

where

$$
\tilde{C}=\left[\begin{array}{ll}
C & R
\end{array}\right], \quad x_{a}=\left[\begin{array}{c}
x_{f} \\
f
\end{array}\right], e \quad{ }_{a}=x_{a}-\hat{x}_{a}
$$

The dynamic of the trajectory tracking error $e=x-x_{f}$, obeys to the differential equation

$$
\begin{aligned}
\dot{e} & =\sum_{i=1}^{r} \mu_{i}(x)\left(A_{i} x+B_{i} u\right) \\
& -\mu_{i}\left(x_{f}\right)\left(A_{i} x_{f}+B_{i}\left(u_{f}-f\right)\right)
\end{aligned}
$$

Taking into account the definitions (6) and (12) leads to

$$
\begin{aligned}
\dot{e} & =\sum_{i=1}^{r} \sum_{j=1}^{r} \mu_{i}\left(x_{f}\right) \mu_{j}\left(\hat{x}_{f}\right)\left(A_{i} e-B_{i}(f-\hat{f})\right. \\
& \left.-B_{i} K_{1 j}\left(x_{f}-\hat{x}_{f}\right)\right)+\Delta_{1} \\
& =\sum_{i=1}^{r} \sum_{j=1}^{r} \mu_{i}\left(x_{f}\right) \mu_{j}\left(\hat{x}_{f}\right)\left(\left(A_{i}-B_{i} K_{1 j}\right) e-\tilde{L}_{i j} e_{a}\right) \\
& +\Delta_{1}
\end{aligned}
$$


where

$$
\begin{aligned}
& \tilde{L}_{i j}=\left(\begin{array}{cc}
B_{i} K_{1 j} & B_{i}
\end{array}\right), \quad e_{a}=x_{a}-\hat{x}_{a} \\
& \Delta_{1}=\sum_{i=1}^{r}\left(\mu_{i}(x)-\mu_{i}\left(x_{f}\right)\right)\left(A_{i} x+B_{i} u\right)
\end{aligned}
$$

In order to analyze the evolution of the errors, two cases are considered : in the first one the faults are supposed to be constant; in the second one they are assumed to be under a polynomial form with respect to the time variable.

\section{A. Constant faults}

In this first approach, we have $\dot{f}(t)=0$ and, with definition (11), the system (5) becomes in augmented form

$$
\left\{\begin{aligned}
\dot{x}_{a} & =\sum_{i=1}^{r} \mu_{i}\left(x_{f}\right)\left(\tilde{A}_{i} x_{a}+\tilde{B}_{i} u_{f}\right) \\
y_{f} & =\tilde{C} x_{a}
\end{aligned}\right.
$$

where

$$
\begin{gathered}
\tilde{A}_{i}=\left(\begin{array}{cc}
A_{i} & B_{i} \\
0 & 0
\end{array}\right), \quad \tilde{B}_{i}=\left(\begin{array}{c}
B_{i} \\
0
\end{array}\right), \\
\tilde{C}=\left(\begin{array}{ll}
C & R
\end{array}\right)
\end{gathered}
$$

The state and fault estimation error $e_{a}(t)=x_{a}(t)-\hat{x}_{a}(t)$ between the system (17) and the observer (7)-(8) evolves according to the following equation

$$
\dot{e}_{a}=\sum_{i=1}^{r} \mu_{i}\left(\hat{x}_{f}\right)\left(\left(\tilde{A}_{i}-H_{i} \tilde{C}\right) e_{a}+\Gamma \Delta_{2}\right)
$$

where

$$
\begin{gathered}
\Gamma=\left[\begin{array}{c}
I_{n} \\
0
\end{array}\right] \\
\Delta_{2}=\sum_{i=1}^{r}\left(\mu_{i}\left(x_{f}\right)-\mu_{i}\left(\hat{x}_{f}\right)\right)\left(A_{i} x_{f}+B_{i}\left(u_{f}+f\right)\right)
\end{gathered}
$$

The concatenation of the state tracking trajectory error and the state and faults estimation errors allows to write, from (13) and (20), the new augmented system is

$$
\dot{\tilde{e}}=\sum_{i=1}^{r} \sum_{j=1}^{r} \mu_{i}\left(x_{f}\right) \mu_{j}\left(\hat{x}_{f}(t)\right) \tilde{A}_{i j} \tilde{e}+\tilde{\Gamma} \Delta
$$

where

$$
\begin{gathered}
\tilde{e}=\left(\begin{array}{c}
x-x_{f} \\
x_{f}-\hat{x}_{f} \\
f-\hat{f}
\end{array}\right), \Delta=\left(\begin{array}{c}
\Delta_{1} \\
\Delta_{2}
\end{array}\right) \\
\tilde{\Gamma}=\left(\begin{array}{cc}
I_{n} & 0 \\
0 & I_{n} \\
0 & 0
\end{array}\right) \\
\tilde{A}_{i j}=\left(\begin{array}{ccc}
A_{i}-B_{i} K_{1 j} & -B_{i} K_{1 j} & -B_{i} \\
0 & A_{j}-H_{1 j} C & B_{j}-H_{1 j} R \\
0 & -H_{2 j} C & -H_{2 j} R
\end{array}\right)
\end{gathered}
$$

The gains $K_{1 i}, H_{1 i}$ and $H_{2 i}$ are determined by solving a minimization problem under LMI constraints, given by the following theorem 1 .

Theorem 1: The state tracking error $e(t)$ and the state and fault estimation errors $e_{a}(t)$ converge asymptotically toward zero if there exists symmetric and positive definite matrices $X_{1}, P_{2}$ and matrices $\bar{H}_{1 i}, H_{2 i}$ and $K_{1 i}$ such that $\bar{\gamma}$ is minimized under the LMI constraints (27).

$$
\begin{gathered}
\left(\begin{array}{ccccccc}
\Psi_{i} & -B_{1} K_{1 j} & -B_{i} & -B_{1} K_{1 j} & X_{1} & X_{1} & 0 \\
* & \Theta_{j} & \Xi_{j} & 0 & 0 & 0 & P_{2} \\
* & * & \Phi_{j} & 0 & 0 & 0 & 0 \\
* & * & * & -I & 0 & 0 & 0 \\
* & * & * & * & -I & 0 & 0 \\
* & * & * & * & * & -\bar{\gamma} I & 0 \\
* & * & * & * & * & * & -\bar{\gamma} I
\end{array}\right)<0 \\
\Psi_{i}=A_{i} X_{1}+X_{1} A_{i}^{T} \\
\Theta_{j}=P_{2} A_{j}+A_{j}^{T} P_{2}-\bar{H}_{1 j} C-C^{T} \bar{H}_{1 j}^{T} \\
\Xi_{j}=P_{2} B_{j}-\bar{H}_{1 j} R-C^{T} \bar{H}_{2 j}^{T} \\
\Phi_{j}= \\
\quad-\bar{H}_{2 j} R-R^{T} \bar{H}_{2 j}^{T} \\
i, j=1, \ldots, r
\end{gathered}
$$

The gains are given by $K_{1 j}$ and $H_{2 j}$ are obtained directly from the above optimization problem and $H_{1 j}$ are then computed from

$$
H_{1 j}=P_{2}^{-1} \bar{H}_{1 j}
$$

the $\mathcal{L}_{2}$ gain from $\Delta$ to $\tilde{e}$ is given by

$$
\gamma=\sqrt{\bar{\gamma}}
$$

Proof: The gains $H_{1 i}, H_{2 i}$ and $K_{1 i}$ are obtained by a stability analysis of the system described by the differential equation (23), using the Lyapunov theory with a quadratic function.

Let us chose the following quadratic Lyapunov function

$$
V(\tilde{e})=\tilde{e}^{T} P \tilde{e}, \quad P=P^{T}>0
$$

where $P$ is chosen as follows

$$
P=\left(\begin{array}{ccc}
P_{1} & 0 & 0 \\
0 & P_{2} & 0 \\
0 & 0 & P_{3}
\end{array}\right)
$$

The time derivative of the function $V(\tilde{e})$ is given by

$$
\begin{aligned}
\dot{V}(\tilde{e}) & =\sum_{i=1}^{r} \sum_{j=1}^{r} \mu_{i}\left(x_{f}\right) \mu_{j}\left(\hat{x}_{f}\right) \tilde{e}^{T}\left(\tilde{A}_{i j}^{T} P+P \tilde{A}_{i j}\right) \tilde{e} \\
& +2 P \tilde{\Gamma} \Delta \\
& =\sum_{i=1}^{r} \sum_{j=1}^{r} \mu_{i}\left(x_{f}\right) \mu_{j}\left(\hat{x}_{f}\right) \tilde{e}^{T} \mathcal{M}_{i j} \tilde{e}+2 P \tilde{\Gamma} \Delta
\end{aligned}
$$

where

$$
\begin{gathered}
\mathcal{M}_{i j}=\mathbb{S}\left(\left(\begin{array}{ccc}
\Lambda_{i j} & -P_{1} B_{i} K_{1 j} & -P_{1} B_{i} \\
0 & \Theta_{j} & \Sigma_{j} \\
0 & -P_{3} H_{2 j} C & -P_{3} H_{2 j} R
\end{array}\right)\right) \\
\Lambda_{i j}=P_{1} A_{i}-P_{1} B_{i} K_{1 j}
\end{gathered}
$$




$$
\begin{gathered}
\Theta_{j}=P_{2} A_{j}-P_{2} H_{1 j} C \\
\Sigma_{j}=P_{2} B_{j}-P_{2} H_{1 j} R
\end{gathered}
$$

and $\mathbb{S}$ is a function that acts on any matrix $X$ as follows

$$
\mathbb{S}(X)=X^{T}+X
$$

Assume that the input and the faults are bounded and that the system is stable. As a consequence, $\Delta((24),(16),(22))$ is bounded. So, the objective is to minimize the $\mathcal{L}_{2}$-gain of $\Delta$ on the error $\tilde{e}(t)$, this is formulated by

$$
\frac{\|\tilde{e}\|_{2}}{\|\Delta\|_{2}}<\gamma, \quad\|\Delta\|_{2} \neq 0
$$

Then, we are seeking to ensure asymptotic convergence of $\tilde{e}(t)$ toward zero if $\Delta(t)=0$ and to guarantee a bounded $\mathcal{L}_{2}$ if $\Delta(t) \neq 0$. This problem can be formulated as follows

$$
\dot{V}(\tilde{e})+\tilde{e}^{T} \tilde{e}-\gamma^{2} \Delta^{T} \Delta<0
$$

After some calculation and by using the convex sum property of the weighting functions, the time varying inequality (43) is satisfied if the following conditions hold

$$
\mathcal{N}_{i j}<0, \quad i, j=1, \ldots, r
$$

where

$$
\mathcal{N}_{i j}=\mathbb{S}\left(\left(\begin{array}{ccccc}
\Lambda_{i j} & -P_{1} B_{i} K_{1 j} & -P_{1} B_{i} & P_{1} & 0 \\
0 & \Theta_{j} & \Sigma_{j} & 0 & P_{2} \\
0 & -P_{3} H_{2 j} C & -P_{3} H_{2 j} R & 0 & 0 \\
P_{1} & 0 & 0 & -\gamma^{2} I & 0 \\
P_{1} & 0 & 0 & 0 & -\gamma^{2} I
\end{array}\right)\right)
$$

by congruence (lemma 2), for every invertible matrix $W$, we have

$$
\mathcal{N}_{i j}<0 \Leftrightarrow W \mathcal{N}_{i j} W<0
$$

defining $W$ by

$$
W=\left(\begin{array}{cccc}
P_{1}^{-1} & 0 & 0 & 0 \\
0 & I & 0 & 0 \\
0 & 0 & I & 0 \\
0 & 0 & 0 & I
\end{array}\right)
$$

the inequality (44) is equivalent to

$$
\left(\begin{array}{ccccc}
\Psi_{i j} & -B_{i} K_{1 j} & -B_{i} & P_{1} & 0 \\
* & Z_{j} & \Upsilon_{j} & 0 & P_{2} \\
* & * & T_{j} & 0 & 0 \\
* & * & * & -\gamma^{2} I & 0 \\
* & * & * & * & -\gamma^{2} I
\end{array}\right)<0
$$

where

$$
\begin{aligned}
\Psi_{i j} & =A_{i} X_{1}+X_{1} A_{i}^{T}-B_{i} K_{1 j} X_{1}-X_{1} K_{1 j}^{T} B_{i}^{T}(49) \\
Z_{j} & =P_{2} A_{j}+A_{j}^{T} P_{2}-P_{2} H_{1 j} C-C^{T} H_{1 j}^{T} P_{2} \\
\Upsilon_{j} & =P_{2} B_{j}-P_{2} H_{1 j} R-C^{T} H_{2 j}^{T} P_{3} \\
T_{j} & =-P_{3} H_{2 j} R-R^{T} H_{2 j}^{T} P_{3} \\
& X_{1}=P_{1}^{-1}
\end{aligned}
$$

Let us remark that the bloc matrix

$$
\left(\begin{array}{ccc}
\Psi_{i j} & -B_{i} K_{1 j} & -B_{i} \\
* & Z_{j} & \Upsilon_{j} \\
* & * & T_{j}
\end{array}\right)
$$

of (48) can be written as follows

$$
\begin{aligned}
& \left(\begin{array}{ccc}
\Psi_{i} & -B_{1} K_{1 j} & -B_{i} \\
* & Z_{j} & \Upsilon_{j} \\
* & * & T_{j}
\end{array}\right) \\
+ & \left(\begin{array}{c}
-B_{i} K_{1 j} \\
0 \\
0
\end{array}\right)\left(\begin{array}{c}
X_{1} \\
0 \\
0
\end{array}\right)^{T}+\left(\begin{array}{c}
X_{1} \\
0 \\
0
\end{array}\right)\left(\begin{array}{c}
-B_{i} K_{1 j} \\
0 \\
0
\end{array}\right)^{T}<0
\end{aligned}
$$

where

$$
\Psi_{i}=A_{i} X_{1}+X_{1} A_{i}^{T}
$$

The lemma 1 gives

$$
\begin{aligned}
& \left(\begin{array}{ccc}
\Psi_{i}-B_{1} K_{1 j} & -B_{i} \\
* & Z j & \Upsilon_{i} \\
* & * & T_{i}
\end{array}\right) \\
+ & \left(\begin{array}{c}
-B_{i} K_{1 j} \\
0 \\
0
\end{array}\right) \Omega^{-1}\left(\begin{array}{c}
-B_{i} K_{1 j} \\
0 \\
0
\end{array}\right){ }^{T} \\
+ & \left(\begin{array}{c}
X_{1} \\
0 \\
0
\end{array}\right) \Omega\left(\begin{array}{c}
X_{1} \\
0 \\
0
\end{array}\right)^{T}<0
\end{aligned}
$$

where $\Omega$ is a symmetric and positive matrix. After bounding the inequality (48) with (57), and, assuming that

$$
\begin{aligned}
\bar{H}_{1 i} & =P_{2} H_{1 i} \\
\bar{\gamma} & =\gamma^{2} \\
\Omega & =I, \quad P_{3}=I
\end{aligned}
$$

the LMIs in theorem 1 are obtained.

\section{B. Time varying faults}

The assumption that the fault signal is constant over the time is restrictive, but in many practical situations where the faults are slowly time-varying signals, the estimation of the faults is correct, and the proposed FTC scheme can be applied. In the case where the faults are not slowly timevarying or constant, the Proportional Integral Observer (PIO) can be replaced by a Proportional Multiple Integral Observer (PMIO). Such an observer is able to estimate a large class of time-varying signals satisfying the following assumption

$$
f^{(q+1)}=0
$$

The principle of this observer is based on the estimation of all the first $q^{t h}$ derivatives of the signal $f(t)$. This observer can also be extended to the case where $f^{(q+1)}$ is bounded.

Let consider the system (5) with a fault in the general polynomial form

$$
f(t)=a_{0}+a_{1} t+a_{2} t^{2}+\ldots+a_{q} t^{q}
$$


Let consider $d_{0}(t)=\dot{f}(t), \quad d_{1}(t)=\ddot{f}(t), \ldots, \quad d_{q-1}(t)=$ $f^{(q)}(t)$, the system can be transformed into an augmented form

$$
\left\{\begin{array}{l}
\dot{\tilde{x}}_{f}=\sum_{i=1}^{r} \mu_{i}\left(x_{f}\right)\left(\tilde{A}_{i} \tilde{x}_{f}+\tilde{B}_{i} u_{f}\right) \\
y=\tilde{C} \tilde{x}_{f}
\end{array}\right.
$$

where

$$
\begin{gathered}
\tilde{x}_{f}=\left(\begin{array}{c}
x_{f} \\
d_{0} \\
\vdots \\
d_{q} \\
d_{(q-1)}
\end{array}\right), \tilde{A}_{i}=\left(\begin{array}{ccccc}
A_{i} & B_{i} & 0 & 0 & 0 \\
0 & 0 & I & 0 & 0 \\
\vdots & \vdots & \vdots & \ddots & \vdots \\
0 & 0 & 0 & 0 & I \\
0 & 0 & 0 & 0 & 0
\end{array}\right), \\
\tilde{B}_{i}=\left(\begin{array}{c}
B_{i} \\
0 \\
\vdots \\
0 \\
0
\end{array}\right), \tilde{C}=\left(\begin{array}{lllll}
C & R & 0 & 0 & 0
\end{array}\right)
\end{gathered}
$$

$\tilde{x}_{f}(t)$ represents the augmented state vector composed of the state $x_{f}(t)$ and the $q^{t h}$ first successive derivatives of the fault $f(t)$. The observer simultaneously estimating the state $x_{f}(t)$ and the faults $f(t)$ with the successive derivatives is given in the following form

$$
\left\{\begin{array}{l}
\dot{\tilde{\tilde{x}}_{f}}=\sum_{i=1}^{r} \mu_{i}\left(\hat{x}_{f}\right)\left(\tilde{A}_{i} \hat{\tilde{x}}_{f}+\tilde{B}_{i} u_{f}+\tilde{H}_{i}(y-\hat{y})\right) \\
\hat{y}=\tilde{C} \tilde{\tilde{x}}_{f}
\end{array}\right.
$$

The augmented state estimation error $e(t)=\tilde{x}_{f}(t)-\hat{\tilde{x}}_{f}(t)$ and the error between $x_{f}$ and $x$ are given by

$$
\left(\begin{array}{c}
\dot{e} \\
\dot{e}_{a}
\end{array}\right)=\sum_{i=1}^{r} \sum_{j=1}^{r} \mu_{i}(x) \mu_{j}\left(\hat{x}_{f}\right) \tilde{\bar{A}}_{i j}\left(\begin{array}{c}
e \\
e_{a}
\end{array}\right)+\tilde{\Gamma} \Delta
$$

where

$$
\tilde{\bar{A}}_{i j}=\left(\begin{array}{cc}
A_{i}-B_{i} K_{1 j} & \tilde{L}_{i j} \\
0 & \tilde{A}_{j}-\tilde{H}_{j} \tilde{C}
\end{array}\right)
$$

Thus, the structure of the state equations is the same as those expressed in the case of constant faults. The synthesis of the gains of the controller and those of the observer are obtained by solving the LMIs given in the theorem 1 .

\section{Simulation example}

The proposed fault tolerant control strategy is illustrated on an academic T-S system described by

$$
\left\{\begin{aligned}
\dot{x}_{f} & =\sum_{i=1}^{r} \mu_{i}\left(x_{f}\right)\left(A_{i} x_{f}+B_{i}\left(u_{f}+f\right)\right) \\
y_{f} & =C x_{f}+R f
\end{aligned}\right.
$$

where

$$
\begin{gathered}
A_{1}=\left[\begin{array}{ccc}
-2 & 1 & 1 \\
1 & -3 & 0 \\
2 & 1 & -8
\end{array}\right], A_{2}=\left[\begin{array}{ccc}
-3 & 2 & 2 \\
0 & -3 & 0 \\
5 & 2 & -4
\end{array}\right], \\
B_{1}=\left[\begin{array}{c}
0 \\
1 \\
0.25
\end{array}\right], B_{2}=\left[\begin{array}{l}
1 \\
1 \\
0
\end{array}\right],
\end{gathered}
$$

$$
C=\left[\begin{array}{lll}
1 & 1 & 1 \\
1 & 0 & 1
\end{array}\right], \quad R=\left[\begin{array}{l}
1 \\
0
\end{array}\right]
$$

The weighting functions depend on the first component of the state vector $x_{f}$; they are defined by

$$
\left\{\begin{array}{l}
\mu_{1}\left(x_{f}\right)=\frac{1-\tanh \left(x_{f}^{1}\right)}{2}\left(x_{f}\right) \\
\mu_{2}\left(x_{f}\right)=1-\mu_{1}\left(x^{\prime}\right)
\end{array}\right.
$$

The input variation $u(t)$ over the time is depicted in the figure 2 (bottom, continuous blue line). To apply the proposed FTC strategy, the following reference model is considered

$$
\left\{\begin{aligned}
\dot{x} & =\sum_{i=1}^{r} \mu_{i}(x)\left(A_{i} x+B_{i} u\right) \\
y & =C x
\end{aligned}\right.
$$

The fault $f(t)$ is a time varying signal at $t=5$. Solving the LMIs in theorem 1 results in the following matrices

$$
\begin{gathered}
X_{1}=\left[\begin{array}{ccc}
0.91 & 0.11 & 0.04 \\
0.11 & 0.93 & -0.04 \\
0.04 & -0.04 & 0.44
\end{array}\right], \\
P_{2}=\left[\begin{array}{ccc}
1.53 & -0.31 & 0.50 \\
-0.31 & 3.04 & -0.39 \\
0.50 & -0.39 & 0.95
\end{array}\right], \\
H_{11}=\left[\begin{array}{cc}
-1.93 & 4.58 \\
-3.19 & 6.27 \\
-5.35 & 1.22
\end{array}\right], \quad H_{12}=\left[\begin{array}{cc}
-3.39 & 5.12 \\
-3.27 & 6.67 \\
-4.47 & 2.74
\end{array}\right], \\
H_{21}=\left[\begin{array}{lll}
4.885 & 0.000
\end{array}\right], H_{22}=\left[\begin{array}{ll}
3.771 & 1.114
\end{array}\right], \\
K_{11}=\left[\begin{array}{lll}
0.004 & 0.024 & -0.004
\end{array}\right], \\
K_{12}=\left[\begin{array}{lll}
0.003 & 0.019 & -0.004
\end{array}\right]
\end{gathered}
$$

The proportional-integral observer provides estimated state and faults. In the figure 2 (top) the real fault and its estimate are depicted. The state estimation errors (resp. the state tracking errors) are displayed on the top (resp. bottom) of figure 3 . The figures 4 compares the state variables of the reference model, of the faulty system without FTC and the faulty system with FTC. One can see that the state variables of the system affected by fault with FTC is closed to the reference whereas the faulty system with nominal control deviates.

\section{CONCLUSION}

This paper is dedicated to the design of a nonlinear fault tolerant control law. The considered systems are modeled in the Takagi-Sugeno fuzzy structure with unmeasurable premise variables. The strategy is based on the use of a reference model which is the model of the system in the fault-free case. The proposed control law is then designed to minimize the deviation of the system state compared to the reference state, even in the presence of fault(s). This control law uses the nominal control input developed for the system in fault-free case and two additional terms. The first term is related to the estimated fault and the second one corresponds to the trajectory tracking error. The stability is studied with the Lyapunov theory and a quadratic function that allows to 

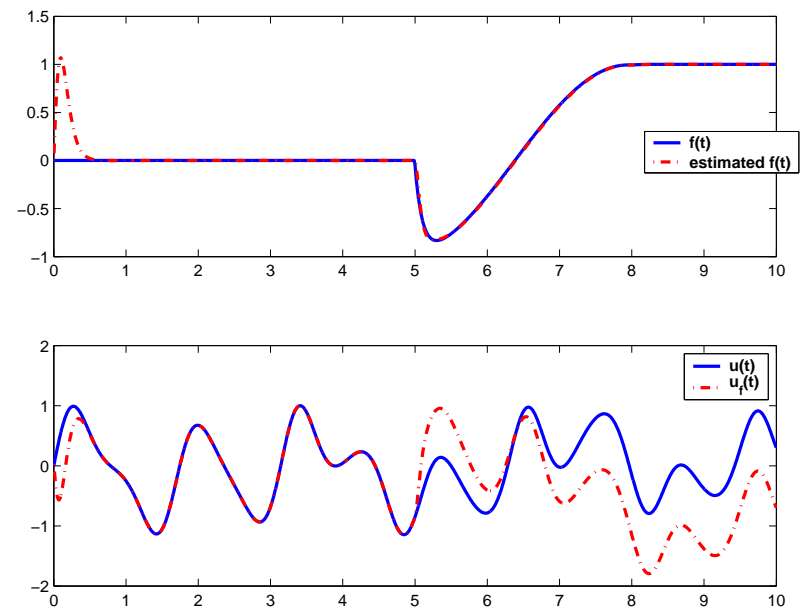

Fig. 2. Fault and its estimate (top) - nominal control input (continuous blue line) FTC input (dashed red line) (bottom)
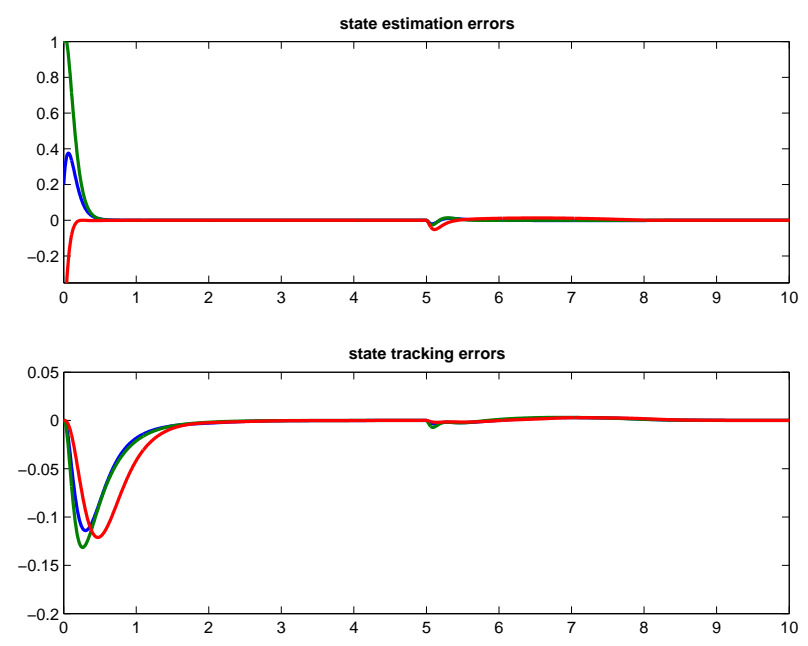

Fig. 3. State estimation errors (top) - State trajectory tracking (bottom)
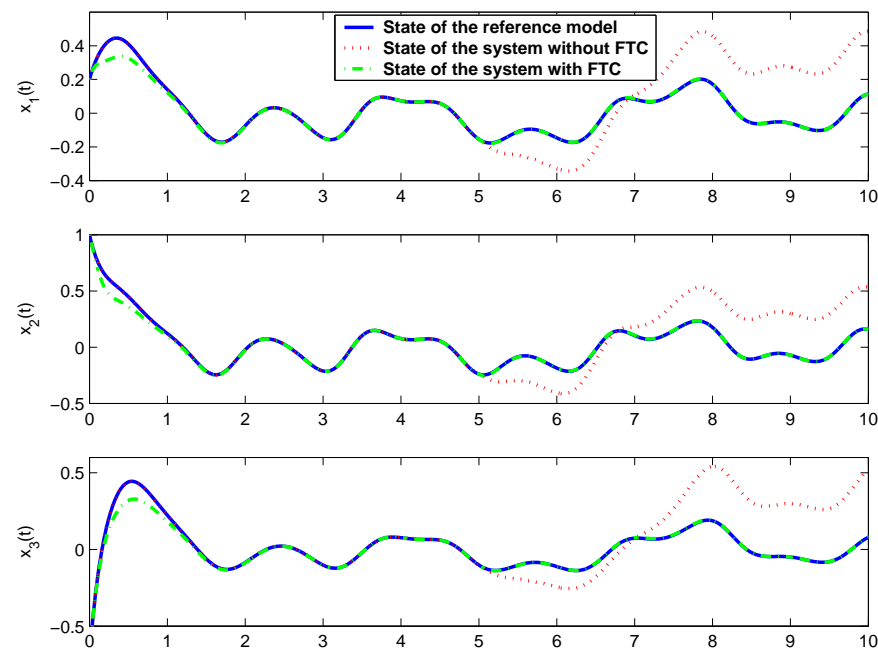

Fig. 4. Comparison between the states of the reference model (no fault), the states of the system with fault and without FTC and states of the system with FTC derive conditions ensuring the convergence of the state and fault estimation errors and trajectory tracking error toward zero. The existence conditions are expressed in terms of LMI that can be solved with classical dedicated softwares. The future works may be oriented, on the one hand, to the relaxation of these conditions by using polyquadratic or non-quadratic Lyapunov functions. On the other hand, the assumption of open-loop stability (needed for solving the LMI problem given in theorem 1) should be relaxed. In addition, it is interesting to develop the FTC control law by taking into account modeling uncertainties, multiplicative faults and some external perturbations, and considering nonlinear outputs of the system. Real applications will be developed in future works.

\section{REFERENCES}

[1] A. Akhenak, M. Chadli, J. Ragot, and D. Maquin. Fault detection and isolation using sliding mode observer for uncertain Takagi-Sugeno fuzzy model. In 16th Mediterranean Conference on Control and Automation Congress Centre, Ajaccio, France, June 25-27 2008.

[2] M. Chadli, D. Maquin, and J. Ragot. Non quadratic stability analysis of Takagi-Sugeno systems. In IEEE Conference on Decision and Control, CDC'2002, Las Vegas, Nevada, USA, 2002.

[3] M. Darouach, M. Zasadzinski, and S.J. Xu. Full-order observers for linear systems with unknown inputs. IEEE Transactions on Automatic Control, 39(3):606-609, March 1994.

[4] Z. Gao and P.J. Antsaklis. Reconfigurable control system design via perfect model following. International Journal of Control, 56(4):783798, 1992.

[5] Z. Gao and S. X. Ding. Actuator fault robust estimation and faulttolerant control for a class of nonlinear descriptor systems. Automatica, 43(5):912-920, 2007.

[6] T-M. Guerra, A. Kruszewski, L. Vermeiren, and H. Tirmant. Conditions of output stabilization for nonlinear models in the TakagiSugeno's form. Fuzzy Sets and Systems, 157(9):1248-1259, May 2006.

[7] A. Kruszewski, R. Wang, and T.M. Guerra. Nonquadratic stabilization conditions for a class of uncertain nonlinear discrete time TS fuzzy models: A new approach. IEEE Transactions on Automatic Control, 53(2):606 - 611, March 2008.

[8] D.G. Luenberger. An introduction to observers. IEEE Transactions on Automatic Control, 16:596-602, 1971.

[9] B. Marx, D. Koenig, and D. Georges. Robust fault tolerant control for descriptor systems. IEEE Transactions on Automatic Control, 49:1869-1875, 2004.

[10] B. Marx, D. Koenig, and J. Ragot. Design of observers for takagi sugeno descriptor systems with unknown inputs and application to fault diagnosis. IET Control Theory and Application, 1:1487-1495, 2007.

[11] M.M. Mufeed, J Jiang, and Z. Zhang. Active Fault Tolerant Control Systems : Stochastic Analysis and Synthesis. Springer, 2003.

[12] A.M. Nagy, G. Mourot, B. Marx, G. Schutz, and J. Ragot. Model structure simplification of a biological reactor. In 15th IFAC Symposium on System Identification, SYSID'09, Saint Malo, France, 2009.

[13] H. Noura, D. Sauter, F. Hamelin, and D. Theilliol. Fault-tolerant control in dynamic systems: Application to a winding machine. IEEE Control Systems Magazine, 20(1):33-49, 2000.

[14] M. Oudghiri. Commande multimodles tolérante aux défauts : application au contrôle de la dynamique d'un véhicule automobile. $\mathrm{PhD}$ thesis, Université de Picardie Jules Verne, Amiens, France, 2008.

[15] M. Staroswiecki. Fault tolerant control : the pseudo-inverse method revisited. In 16th IFAC World Congress, Seoul, Korea, 2005.

[16] T. Takagi and M. Sugeno. Fuzzy identification of systems and its applications to modeling and control. IEEE Transactions on Systems, Man, and Cybernetics, 15:116-132, 1985.

[17] K. Tanaka and H.O. Wang. Fuzzy Control Systems Design and Analysis: A Linear Matrix Inequality Approach. John Wiley and Sons, 2001. 